\title{
BMJ Open Diabetes-related symptoms, acute complications and management of diabetes mellitus of patients who are receiving palliative care: a protocol for a systematic review
}

\author{
Rita Bettencourt-Silva, ${ }^{1,2}$ Beatriz Aguiar, ${ }^{3}$ Vânia Sá-Araújo, ${ }^{4}$ Rosa Barreira, ${ }^{5}$ \\ Vânia Guedes, ${ }^{6}$ Maria João Marques Ribeiro, ${ }^{7}$ Davide Carvalho, ${ }^{2,8}$ Linda Östlundh, ${ }^{9}$ \\ Marília Silva Paulo 10,11
}

To cite: Bettencourt-Silva $R$, Aguiar B, Sá-Araújo V, et al. Diabetes-related symptoms, acute complications and management of diabetes mellitus of patients who are receiving palliative care: a protocol for a systematic review. BMJ Open 2019;9:e028604. doi:10.1136/ bmjopen-2018-028604

- Prepublication history and additional material for this paper are available online. To view these files, please visit the journal online (http://dx.doi org/10.1136/bmjopen-2018028604).

Received 18 December 2018 Revised 1 May 2019 Accepted 28 May 2019

Check for updates

(C) Author(s) (or their employer(s)) 2019. Re-use permitted under CC BY-NC. No commercial re-use. See rights and permissions. Published by BMJ.

For numbered affiliations see end of article.

Correspondence to

Marília Silva Paulo;

mariliap@uaeu.ac.ae

\section{ABSTRACT}

Introduction Worldwide, an estimated 40 million people are in need of palliative care each year, but only $14 \%$ receive it. The incidence of diabetes mellitus (DM) in patients receiving palliative care is higher than in the general population. This association is intended to grow as a result of the rising burden of DM worldwide, ageing populations and the improved overall survival time of several diseases over the last few decades. Recommendations for DM management in the context of palliative care are mainly based on expert opinion as there is a lack of suitable evidence base and randomised clinical trials in palliative care are scarce. The aim of our systematic review is to identify the best DM management practices in order to reduce important DM-related symptoms and acute complications in patients receiving palliative care.

Methods and analysis The authors will study the DM treatment and management literature, surveying the different approaches employed to treat adult palliative patients. Core health bibliographic databases will be searched from January 1990 to May 2019. Data sources will include Ovid MEDLINE, Embase, PubMed, Web of Sciences, Cochrane Central Register of Controlled Trials, Cochrane Database of Systematic Reviews, Scopus, Cumulative Index to Nursing and Allied Health Literature and grey literature. Details regarding diet, oral and injectable glucose-lowering medicines, insulin regimens and blood glucose monitoring strategies will be evaluated. We defined the primary outcomes to compare between DM management approaches as the presence of symptoms (polyuria, polydipsia and polyphagia) and acute complications of DM (hypoglycaemia, hyperglycaemic hyperosmolar state and diabetic ketoacidosis), and secondary outcomes as hospital admissions and deaths due to DM-related complications, health-related quality of life and glycaemic control.

Ethics and dissemination The systematic review methodology does not require ethics approval due to the nature of the study design. The results of the systematic review will be published in a peer-reviewed journal and will be publicly available.
Strengths and limitations of this study

The present protocol is an outline of the systematic review specificities that will guide the authors while conducting the systematic review on the diabetes-related symptoms, acute complications and management of diabetes mellitus in palliative care.

- Limitations of this review include the exclusion of papers reported in languages other than English, Portuguese or Spanish and those published prior to 1990.

- The inclusion of grey literature will broaden this study in terms of included information.

- This systematic review will include primary studies of any design because, as far as we know, this is the first systematic review on the diabetes-related symptoms and complications in palliative care.

PROSPERO registration number CRD42018115772.

\section{INTRODUCTION}

WHO defines palliative care as an approach that improves the quality of life (QOL) of patients and their families facing problems associated with life-threatening illness, through the prevention and relief of suffering by means of early identification and impeccable assessment and treatment of pain and other problems, physical, psychosocial and spiritual. ${ }^{1}$ Palliative care can be provided to a person of any age and it is not limited to patients who are actively dying, is appropriate at any stage (stable, unstable, deteriorating or terminal) of serious illnesses (cancer and/or another disease). ${ }^{2}$ Palliative care is best introduced early in the course of illness and it can be provided alongside curative treatment or other therapies that 
are intended to prolong life, such as chemotherapy or radiation therapy. Worldwide, an estimated 40 million people are in need of palliative care each year, but only $14 \%$ receive it. ${ }^{3}$ The majority of adults in need of palliative care have serious diseases such as cardiovascular diseases $(38.5 \%)$, cancer $(34 \%)$ and chronic respiratory diseases $(10.3 \%) .^{3}$

Diabetes mellitus (DM) affects $>400$ million people, corresponding to $8.5 \%$ of adults worldwide. ${ }^{3}$ The incidence of DM in patients receiving palliative care is higher than in the general population due to several factors, such as age, use of diabetogenic drugs such as corticosteroids and metabolic changes due to chronic disease. This association is intended to grow as a result of the rising burden of DM worldwide, ageing populations and the improved overall survival time of several diseases over the last few decades. ${ }^{4}$ The diagnosis of DM has already been made in the majority of patients who are referred to palliative care services. Furthermore, DM is, by itself, an increased cause of palliative enrolment in some countries with advanced health system integration as it can be considered as a life-threatening illness. ${ }^{5-7}$ Evidencebased practice guidelines for the management of DM have been developed by several scientific associations, ${ }^{8-12}$ which highlight that nutrition, pharmacological therapy, self-monitoring blood glucose and glycated haemoglobin (HbAlc) targets should be individualised for each patient. Additionally, goals should be individualised based on the duration of DM, age or life expectancy, comorbid conditions, known cardiovascular disease or advanced microvascular complications, hypoglycaemia unawareness and individual patient considerations. ${ }^{13}$ Although less restrictive glycaemic targets for DM management in the context of palliative care are usually suggested, recommendations are mainly based on expert opinion. Indeed, there is a lack of relevant evidence particularly from randomised clinical trials that guides DM management in the context of palliative care. Even the opinions of diabetologists and oncologists were different dealing with diabetes care in people with cancer. ${ }^{14}$ To the best of our knowledge, the most resourceful nutritional approach, oral and injectable (non-insulin) agents, types of insulin or insulin regimens, are still unknown to manage DM in the palliative context. Therefore, the DM management in these patients remains a challenge.

Palliative medicine manages the whole person, providing relief from pain, depression and other distressing symptoms at any stage of the disease and treats joint chronic diseases. As such, the appropriate treatment of hyperglycaemia-related symptoms and DM-related acute complications can contribute to worsening patients' general condition and QOL, particularly acute complications, which commonly lead to hospital admissions and additional consequences. Comfort, patient preferences, evaluation of oral intake and preservation of QOL are of particular importance in palliative medicine and interdisciplinary team care and early referral to relevant clinicians are essential.

\section{Objectives}

This systematic review was developed after a multidisciplinary discussion between the authors of shared doubts and concerns regarding DM management in the context of palliative care. The aim of our systematic review is to identify the best management of DM in order to reduce important DM-related symptoms (polyuria, polydipsia and polyphagia) and acute complications (hypoglycaemia, hyperglycaemic hyperosmolar state and diabetic ketoacidosis) in patients receiving palliative care. The results of this systematic review may guide practices to improve patient care and be a stimulus for additional studies regarding DM management in palliative care.

\section{METHODS AND ANALYSIS}

The present protocol followed the preferred reporting items for systematic reviews and meta-analysis protocols (PRISMA-P) that was defined in $2015,{ }^{15}$ subsequent to the PRISMA statement. The protocol is registered online on the international prospective register of systematic reviews, PROSPERO: CRD42018115772.

\section{Eligibility criteria}

The population, intervention, comparator and outcomes as described by the PRISMA statement ${ }^{16}$ are reported below.

\section{Type of population}

We will include studies of adult (aged $\geq 18$ years) patients with diabetes receiving palliative care. We will exclude studies of paediatric population, those who do not have $\mathrm{DM}$ and the patients who are not in palliative care.

\section{Type of intervention}

We will include primary studies reporting the intervention: management of DM. Details regarding diet, oral and injectable (non-insulin) glucose-lowering drugs, insulin regimens and blood glucose monitoring strategies will be evaluated. Different approaches according to the aetiology of DM, duration of DM, stage of palliative disease (stable, unstable, deteriorating, terminal) will also be assessed.

\section{Type of comparator}

The comparator item will be the different treatments and/or approaches used to manage DM in patients receiving palliative care.

\section{Type of outcomes}

We have defined primary outcomes as specific measures of the presence of symptoms (polyuria, polydipsia and polyphagia) and acute complications of DM (hypoglycaemia, hyperglycaemic hyperosmolar state and diabetic ketoacidosis) in order to make a feasible comparison of the different types of DM management approaches. If data exist, we will also report the secondary outcomes: hospital admissions due to DM-related complications, deaths due to DM-related complications, health-related 
QOL (objective measures), advanced care planning, documented advance care plans and glycaemic control measured by HbA1c or average glycaemia.

\section{Type of studies}

We will include studies of any design regarding DM treatment and management in adult patients receiving palliative care. We will include only studies with a minimum of 10 participants. We will exclude editorials, comments, case reports and other reviews and studies before 1990 (year of the first formal definition of palliative care by WHO).

\section{Information sources}

We will search the following electronic bibliographic databases: Ovid MEDLINE, Embase, PubMed Central, Web of Sciences, Cochrane Central Register of Controlled Trials, Cochrane Database of Systematic Reviews, Scopus, Cumulative Index to Nursing and Allied Health Literature. Grey literature will be searched using the Grey Literature Report and Open Grey databases.

\section{Search strategy}

The search will be conducted as soon as this protocol is published. Core health bibliographic databases will be searched from January 1990 to May 2019.

The search strategy will include terms defined in accordance with population, intervention, comparator and outcomes as described above. The search strategy will be defined for PubMed and then will be adapted for use with other bibliographic databases. No filters or limitations will be applied to the search strategy to ensure retrieval of pre-indexed materials. Only studies in English, Spanish and Portuguese will be included. Grey literature will include unpublished conference proceedings or abstracts from relevant specialty conferences. Reference lists of previous reviews and of included studies will also be screened looking for potentially eligible studies. The full search strategy for PubMed is available in the online supplementary material.

\section{Study records}

Data management

We will apply the inclusion and exclusion criteria outlined above to the potentially eligible studies retrieved using our search strategy. For studies selection, we will use Covidence-Better Systematic Review Management. ${ }^{17}$ All the records identified in the search strategy will be downloaded as Research Information Systems files and uploaded to EndNote (as a security copy) and to Covidence.

\section{Selection process}

Covidence will automatically identify and exclude the duplicates. After obtaining the final potentially eligible articles to include in this systematic review, titles and abstracts will be screened by two independent reviewers considering the inclusion and exclusion criteria outlined. Based on these criteria, the independent reviewers will accept or reject studies. Each study will be voted by the two authors. Conflicts will be solved by a third author not involved in screening the papers. Potentially eligible studies will be uploaded into Covidence for full-text screening. Similarly, this process will be done by two independent reviewers. The same procedure will be applied and any disagreement between them will be resolved by a third reviewer.

\section{Data collection process}

Relevant data will be extracted into a predefined data extraction sheet. This data extraction sheet will be designed for the purpose of this study and will be piloted per the authors responsible for the data extraction with a minimum of five studies.

Seven authors will extract data from potentially eligible studies. Each study will be double-reviewed: one author will extract the data and a second author will independently check the data extraction forms for accuracy and detail. Discrepancies between the authors will be solved by another author and discussed until reaching consensus. Data extraction will also be done using the software Covidence. The authors of eligible studies will be contacted by email to provide missing or additional data.

\section{Data items}

The data extraction sheet will contain information regarding study setting, study population, participant's characteristics, study methodology, life-threatening illness-related data (aetiology of disease, stage of palliative care), details of DM (type and duration of DM, usual treatment namely class of oral or injectable (non-insulin) glucose-lowering drugs, type of insulin, insulin regimen), type of DM management used in the palliative care (blood glucose monitoring strategies, diet, oral glucose-lowering medicines, type of insulin and insulin regimen such as sliding scale insulin or scheduled insulin therapy with only basal insulin treatment or basal bolus insulin regimen ${ }^{18}$ and information for assessment of the risk of bias. The frequency of DM-related symptoms (polyuria, polydipsia and polyphagia) and DM-related acute complications (hypoglycaemia, hyperglycaemic hyperosmolar state and diabetic ketoacidosis) will be extracted and evaluated according to different DM approaches, such as insulin treatment alone or combined with other glucose-lowering drugs. Hospital admission and deaths due to DM-related complications, QOL, HbA1c and average glycaemia will also be extracted where available.

\section{Outcomes and prioritisation}

All the variables will be extracted as string variables. As mentioned in the 'Type of outcomes' section, we will extract all the data available regardless if it is a main or additional outcome. We will not apply prioritisation schemes as meta-analysis will not be performed.

Risk of bias in individual studies

Two independent reviewers will assess the risk of bias and quality evidence of the eligible studies using the 
Study Quality Assessment Tools of the National Institutes of Health. ${ }^{19}$ Disagreements between the review authors over the risk of bias will be resolved by a third author and discussed until reaching consensus. Any discrepancies or unusual patterns will be checked with the study investigator, although we will not exclude studies based on their quality report, we will describe the quality of the included studies.

\section{Data synthesis}

The results will be summarised in tables containing the year, country, population, type of treatment and/or approach for DM and the outcomes. The comparison between these variables will be further described. We will write a descriptive synthesis of the findings from the included studies, structured from the patients' characteristics, type of diabetes management, details of the disease for which the patient was referred to palliative care and acute complications and symptoms of DM. If data exist, we will provide summaries of intervention effects for each study.

\section{Analysis of subgroups or subsets}

If there is enough data, we will perform subgroup analysis by age groups, sex, aetiology of the disease requiring palliative care, stage of palliative care, type of DM, different settings (acute or primary care sector, professional or family care) and study design.

\section{Patient and public involvement}

Patients and the public will not be involved in this study.

\section{Ethics and dissemination}

The systematic review methodology does not require ethics approval due to the nature of the study design. The results of the systematic review will be published in a peer-reviewed journal and will be publicly available. It will also be disseminated electronically and in printed versions.

\section{Author affiliations}

${ }^{1}$ Department of Endocrinology, Diabetes and Metabolism, Centro Hospitalar Universitário São João, Porto, Portugal

${ }^{2}$ Faculty of Medicine, Instituto de Investigação e Inovação em Saúde, University of Porto, Porto, Portugal

${ }^{3}$ Unidade de Saúde de llha de São Miguel, Unidade de Saúde de Rabo de Peixe, Ribeira Grande, São Miguel, Açores, Portugal

${ }^{4}$ Department of Palliative Care, Instituto Português de Oncologia do Porto Francisco Gentil, Porto, Portugal

${ }^{5}$ Unidade de Saúde Familiar Maresia, Unidade Local de Saúde de Matosinhos, Matosinhos, Portugal

${ }^{6}$ Unidade de Saúde Familiar São João do Porto, Agrupamento de Centros de Saúde (ACES) do Porto 0cidental, Porto, Portugal

${ }^{7}$ Department of Medical Oncology, Centro Hospitalar Universitário São João, Porto, Portugal

${ }^{8}$ Department of Endocrinology, Diabetes and Metabolism, Centro Hospitalar Universitário de São João, Porto, Portugal

${ }^{9}$ National Medical Library, United Arab Emirates University College of Medicine and Health Sciences, Al Ain, Abu Dhabi, United Arab Emirates

${ }^{10}$ Institute of Public Health, United Arab Emirates University College of Medicine and Health Sciences, Al Ain, United Arab Emirates

${ }^{11}$ Universidade Nova de Lisboa Instituto de Higiene e Medicina Tropical, Lisboa, Portugal
Contributors RB-S is the primary author for the study. RB-S and MSP conceived, designed the study and drafted the protocol. MSP is the corresponding author. LO performed the full search strategy for each database. LÖ provided feedback on study protocol design and critically revised earlier versions of the manuscript. BA, VS-A, RB, VG, MJMR and DC critically revised earlier drafts of the manuscript for intellectual content. All the authors read and approved the final manuscript.

Funding The authors have not declared a specific grant for this research from any funding agency in the public, commercial or not-for-profit sectors.

Competing interests None declared.

Patient consent for publication Not required.

Provenance and peer review Not commissioned; externally peer reviewed.

Open access This is an open access article distributed in accordance with the Creative Commons Attribution Non Commercial (CC BY-NC 4.0) license, which permits others to distribute, remix, adapt, build upon this work non-commercially, and license their derivative works on different terms, provided the original work is properly cited, appropriate credit is given, any changes made indicated, and the use is non-commercial. See: http://creativecommons.org/licenses/by-nc/4.0/.

\section{REFERENCES}

1. World Health Organization. WHO | WHO Definition of Palliative Care. WHO. 2012 http://www.who.int/cancer/palliative/definition/en/ (accessed 14 Nov 2018).

2. World Health Organization. Palliative Care: symptom management and end-of-life care. Integrated Management of Adolescents and Adult IIIness - Interim Guidelines for first level facilityhealth workers. 2004 https://www.who.int/3by5/publications/documents/en/genericp alliativecare082004.pdf (accessed 10 Mar 2019).

3. World Health Organization. WHO Fact Sheets on Palliative Care. http://www.who.int/news-room/fact-sheets/detail/palliative-care (accessed 14 Nov 2018).

4. Surveillance Research Program NCI. SEER*Explorer: An interactive website for SEER cancer statistics. https://seer.cancer.gov/explorer/ (accessed 14 Nov 2018).

5. Johnston GM, Lethbridge L, Talbot P, et al. Identifying persons with diabetes who could benefit from a palliative approach to care. Can $J$ Diabetes 2015;39:29-35.

6. The World Hospice Palliative Care Association. WHO Global Atlas on Palliative Care At the End of Life. http://www.thewhpca.org/ resources/global-atlas-on-end-of-life-care (accessed 14 Nov 2018).

7. Dunning T, Martin P. Palliative and end of life care of people with diabetes: Issues, challenges and strategies. Diabetes Res Clin Pract 2018;143:454-63.

8. American Diabetes Association. 12. Older Adults: Standards of Medical Care in Diabetes-2019. Diabetes Care 2019;42:S139-47.

9. Dunning T, Sinclair A, Colagiuri S. New IDF Guideline for managing type 2 diabetes in older people. Diabetes Res Clin Pract 2014;103:538-40.

10. Davies MJ, D'Alessio DA, Fradkin J, et al. Management of Hyperglycemia in Type 2 Diabetes, 2018. A Consensus Report by the American Diabetes Association (ADA) and the European Association for the Study of Diabetes (EASD). Diabetes Care 2018;41:2669-701.

11. Garber AJ, Abrahamson MJ, Barzilay JI, et al. COnsensus statement by the american association of clinical endocrinologists and american college of endocrinology on the comprehensive type 2 diabetes management algorithm - 2018 executive summary. Endocr Pract 2018;24:91-120.

12. Ferrari $P$, Giardini A, Negri EM, et al. Managing people with diabetes during the cancer palliation in the era of simultaneous care. Diabetes Res Clin Pract 2018;143:443-53.

13. American Diabetes Association. 6. Glycemic Targets: Standards of Medical Care in Diabetes-2019. Diabetes Care 2019;42:S61-70.

14. Clemente G, Giorgini M, Mancini M, et al. Diabetologists and Oncologists attitudes towards treating diabetes in the oncologic patient: Insights from an exploratory survey. Diabetes Res Clin Pract 2018;143:420-7.

15. Shamseer L, Moher D, Clarke M, et al. Preferred reporting items for systematic review and meta-analysis protocols (PRISMA-P) 2015 : elaboration and explanation. BMJ 2015;350:g7647.

16. Liberati A, Altman DG, Tetzlaff J, et al. The PRISMA statement for reporting systematic reviews and meta-analyses of studies that evaluate health care interventions: explanation and elaboration. PLoS Med 2009;6:e1000100.

17. Babineau J. Product Review: Covidence (Systematic Review Software). J Can Heal Libr Assoc / J l'Association des bibliothèques la santé du Canada 2014;35:68. 
18. Umpierrez GE, Hellman R, Korytkowski MT, et al. Management of hyperglycemia in hospitalized patients in non-critical care setting: an endocrine society clinical practice guideline. J Clin Endocrinol Metab 2012;97:16-38.
19. National Heart Lung and Blood Institute. Study Quality Assessment Tools - NHLBI, NIH. 2016 https://www.nhlbi.nih.gov/ health-topics/study-quality-assessment-tools (accessed $21 \mathrm{Nov}$ 2018). 